\begin{tabular}{c} 
International Journal of Advanced Astronomy, 3(2)(2015) $117-122$ \\
International Journal of Advanced Astronomy \\
SPC \\
Journal home page: $\begin{array}{c}\text { www.sciencepubco.com/index.php/IJAA } \\
\text { doi: } 10.14419 / \text { ijaa. } v 3 i 2.5401 \\
\text { Research Paper }\end{array}$ \\
\hline
\end{tabular}

\title{
Scalar field cosmology in Lyra's geometry
}

\author{
V. K. Shchigolev *, E. A. Semenova \\ Department of Theoretical Physics, Ulyanovsk State University, Ulyanovsk, Russia \\ *Corresponding author E-mail: vkshch@yahoo.com
}

\begin{abstract}
The new classes of homogeneous cosmological models for the scalar fields are build in the context of Lyra's geometry. The different types of exact solution for the model are obtained by applying two procedures, viz the generating function method and the first order formalism.
\end{abstract}

Keywords: Cosmology; Lyra's Geometry; Phantom; Scalar Field; Tachyon Field.

\section{Introduction}

After the formulation of General Relativity (GR) by Einstein, many alternative geometric theories have been developed in order to explain gravitation phenomena. Inspired by geometrician gravitation, Weyl (1918) proposed a more general theory in which both gravitation and electromagnetism are described geometrically. For a long time, Weyl's theory was not taken seriously due to nonintegrability of length of vector under parallel displacement. Later, Lyra (1951) suggested a modification of Riemannian geometry by introducing a gauge function which removes the non-integrability condition of the length of a vector under the parallel transport. This modified Riemannian geometry is known as Lyra's geometry. In contrast to Weyl's geometry, in Lyra's geometry, the connection is metric preserving as in Riemannian geometry, and length transfers are integrable. It should be noted that Lyra introduced a gauge function into the structure-less manifold, as a result of which a displacement field arises naturally. Several authors (see, e.g. Sen, 1957: Sen and Dunn, 1971; Beesham, 1986, Pradhan and Yadav, 2009; Shchigolev, 2012) have studied cosmology in Lyra's geometry. This alternating theory is of interest because it produces effects similar to those produced in Einstein's theory.

Soleng (1987) has pointed out that the cosmologies based on Lyra's manifold with constant gauge vector will either include a creation field and be equal to Hoyle's creation field cosmology (Hoyle and Narlikar, 1964), or contain a special vacuum field, which together with the gauge vector term, may be considered as a cosmological term. Contrary to common assertion that the displacement vector field can play the role of the cosmological constant by itself, we want to emphasize that it can never play this role being alone, as seen from the equations presented below in this article. In general relativity, Einstein succeeded in geometrizing gravitation by identifying the metric tensor with the gravitational potentials. In the scalar tensor theory of Brans-Dicke on the other hand, the scalar field remains alien to the geometry. Lyra's geometry is more in keeping with the spirit of Einstein's principle of geometrisation since both the scalar and tensor fields have more or less intrinsic geometrical significance. Furthermore, the present theory predicts the same effects, within observational limits, as far as the classical Solar System tests are concerned, as well as tests based on the linearized form of the field equations (Beesham, 1988).).

To date, several authors have studied cosmology in Lyra's geometry with both a constant displacement field and a time-dependent one. For instance (Beesham, 1988),), the displacement field is allowed to be time dependent, and the Friedmann-RobertsonWalker (FRW) models are derived in Lyra's manifold. Those models are free of the big-bang singularity and solve the entropy and horizon problems which beset the standard models based on Riemannian geometry. Recently, cosmological models in the frame work of Lyra's geometry in different contexts have been investigated in several papers (see, e.g Pradhan et al., 2011; Agarwal, 2011; Chaubey, 2012; Shchigolev, 2013).

In the last few decades there has been considerable interest in alternative theories of gravitation coursed by the investigations of inflation and, especially, late cosmological acceleration which is well proved (Riess et al., 1998; Perlmutter et al.; 1999). In order to explain so unexpected behavior of our universe, one can modify the gravitational theory, or construct various field models of the so-called dark energy (DE) which equation of state (EoS) satisfies $w=p / \rho<-1 / 3$. Presently, there is an uprise of interest in scalar fields in GR and alternative theories of gravitation in this context. Therefore, the study of cosmological scalar-field models in Lyra's geometry may be relevant for the cosmic acceleration models. Most studies in Lyra's cosmology involve a perfect fluid. Strangely, at least up to our knowledge, the case of scalar field in Lyra's cosmology was not studied properly. Here we would like to fill this gap. In this paper, we consider a scalar (quintessence or phantom) field and a tachyon field cosmological evolution in the context of Lyra's geometry. With motivation provided above, we have obtained exact solutions of Einstein's modified field equations for the spatially flat Friedmann metric within the frame work of Lyra's geometry. For this purpose, we employ two methods, viz the generating function method and the first order formalism.

\section{Field equations}

The Einstein field equations based on Lyra's manifold, as proposed by Sen and Dunn (1971) in normal gauge may be written as. 


$$
R_{i k}-\frac{1}{2} g_{i k} R+\frac{3}{2} \varphi_{i} \varphi_{k}-\frac{3}{4} g_{i k} \varphi^{j} \varphi_{j}=-\kappa^{2} T_{i k},
$$

Where $\varphi_{i}$ is the displacement vector, $\kappa^{2}=8 \pi G$ and other symbols have their usual meanings in the Rimannian geometry. We assume a perfect fluid form for the energy-momentum tensor:

$$
T_{i k}=(\rho+p) u_{i} u_{k}-p g_{i k},
$$

And co-moving coordinates $u_{i} u^{i}=1$, where $u_{i}=(1,0,0,0)$. We also let $\varphi_{i}$ be a time - like vector

$\varphi_{i}=(\beta, 0,0,0)$,

Where $\beta=\beta(t)$ is a function of time alone The metric for FRW space-time is given by

$d s^{2}=d t^{2}-a^{2}(t)\left(d r^{2}+\xi^{2}(r) d \Omega^{2}\right)$,

Where $\xi(r)=\sin r, r, \sinh r$ in accordance with a sign of the curvature $k=+1,0,-1$. For this metric together with (2) and (3), the field equations (1) become

$$
3 H^{2}+\frac{3 k}{a^{2}}-\frac{3}{4} \beta^{2}=\kappa^{2} \rho, \text { (5) } 2 \dot{H}+3 H^{2}+\frac{k}{a^{2}}+\frac{3}{4} \beta^{2}=-\kappa^{2} p,
$$

Where $H=\dot{a} / a$ is the Hubble's parameter?

Equations (5) and (6) lead to the continuity equation as follows

$$
\dot{\rho}+\frac{3}{2 \kappa^{2}} \beta \dot{\beta}+3 H\left(\rho+p+\frac{3}{2 \kappa^{2}} \beta^{2}\right)=0 .
$$

It is easy to find that the main equations of the model, i.e. (5), (6) and (7), can be presented in standard GR form,

$$
\begin{aligned}
& 3 H^{2}+\frac{3 k}{a^{2}}=\kappa^{2} \rho_{\text {eff }}, \\
& 2 \dot{H}+3 H^{2}+\frac{k}{a^{2}}=-\kappa^{2} p_{e f f},
\end{aligned}
$$

And

$$
\dot{\rho}_{\text {eff }}+3 H\left(\rho_{\text {eff }}+p_{\text {eff }}\right)=0,
$$

By introducing two effective parameters:

$$
\rho_{\text {eff }}=\rho+\frac{3 \beta^{2}}{4 \kappa^{2}}, \quad p_{e f f}=p+\frac{3 \beta^{2}}{4 \kappa^{2}} .
$$

To study this model, it is suitable to consider the EoS parameter $w$ and deceleration parameter $q$ defined by

$w=\frac{p_{\text {eff }}}{\rho_{\text {eff }}}=-1-\frac{2}{3} \frac{\dot{H}}{H^{2}}, q=-\frac{a \ddot{a}}{\dot{a}^{2}}=-1-\frac{\dot{H}}{H^{2}}$.

In the absence of matter, that is when $\rho=p=0$, the effective EoS is equal to $w=+1$, which corresponds to the so-called stiff fluid. That is why the displacement vector can never play the role of a cosmological term for which the $\operatorname{EoS} w=-1$ is required.
To proceed further, we have to specify the type of scalar field. For the sake of simplicity, from now on we consider a flat FRW cosmology: $k=0$.

\section{Quintessence (phantom) Lyra's cosmology}

In this section, we consider a quintessence (or phantom) field as a source of gravity in Lyra's cosmology. Therefore, we have for the effective parameters (11) as follows

$\rho_{\text {eff }}=\frac{\varepsilon}{2} \dot{\phi}^{2}+V(\phi)+\frac{3 \beta^{2}}{4 \kappa^{2}}$

$p_{\text {eff }}=\frac{\varepsilon}{2} \dot{\phi}^{2}-V(\phi)+\frac{3 \beta^{2}}{4 \kappa^{2}}$,

Where $\varepsilon=+1$ represents quintessence while $\varepsilon=-1$ refers to phantom field. In view of (13), the set of basic equations (8), (9) becomes

$3 H^{2}=\varepsilon \dot{\phi}^{2}+2 V(\phi)+\frac{3}{4} \beta^{2}$,

$\dot{H}=-\varepsilon \dot{\phi}^{2}-\frac{3}{4} \beta^{2}$,

Where and in what follows we assume $4 \pi G=1$. Even for the given potential $V(\phi)$ and displacement function $\beta(t)$, it is difficult to find exact solution for this model. However, a class of exact solutions can be obtained, say, in terms of the so-called generating function (Zhuravlev and Chervon, 2000).

\subsection{The generating function method}

Since $\beta(t)$ is an arbitrary function so far, we can transfer this arbitrariness into a new one, say coupling function $f(\phi(t))$ as follows

$$
\beta^{2}=\frac{4}{3} f^{2}(\phi) \dot{\phi}^{2}
$$

As a result, we have from (13) and (16) that

$\rho_{e f f}=\frac{1}{2}\left[f^{2}(\phi)+\varepsilon\right] \dot{\phi}^{2}+V(\phi)$,

$p_{\text {eff }}=\frac{1}{2}\left[f^{2}(\phi)+\varepsilon\right] \dot{\phi}^{2}-V(\phi)$.

Re-defying the scalar field as $\left|f^{2}(\phi)+\varepsilon\right| \dot{\phi}^{2}=\dot{\psi}^{2}$, i.e.

$\psi= \pm \int \sqrt{\left|f^{2}(\phi)+\varepsilon\right|} d \phi$,

And putting $\varepsilon=\operatorname{sgn}\left[f^{2}(\phi)+\varepsilon\right]$,we get the following expressions for the effective parameters (13):

$\rho_{\text {eff }}=\frac{\varepsilon}{2} \dot{\psi}^{2}+U(\psi), \quad p_{\text {eff }}=\frac{\varepsilon}{2} \dot{\psi}^{2}-U(\psi)$

Where $U(\psi)=V(\phi(\psi))$. As seen in the effective parameters, the sign $\varepsilon$ of kinetic term can change (for $\varepsilon=-1$ ) from positive to negative and vice versa. Substituting (17) and (18) into Eqs. (8) and (9), we have the following set of the main equations for our model: 


$$
\begin{aligned}
& H^{2}=\frac{2}{3}\left(\frac{1}{2}\left[f^{2}(\phi)+\varepsilon\right] \dot{\phi}^{2}+V(\phi)\right), \\
& \dot{H}=-\left[f^{2}(\phi)+\varepsilon\right] \dot{\phi}^{2} .
\end{aligned}
$$

In order to obtain exact solutions and following to the method of generating function, we assume that $F(\phi)=\dot{\phi}$. Applying the latter to the set of equations (19), (20), we can obtain the following general solution:

$$
t(\phi)=\int \frac{d \phi}{F(\phi)},
$$

$H(\phi)=-\int\left[f^{2}(\phi)+\varepsilon\right] F(\phi) d \phi$,

$V(\phi)=\frac{3}{2} H^{2}(\phi)-\frac{1}{2}\left[f^{2}(\phi)+\varepsilon\right] F^{2}(\phi)$,

And

$$
a(\phi)=a_{0} \exp \left(\int \frac{H(\phi)}{F(\phi)} d \phi\right)
$$

Moreover, as it follows from (12) and (22), the EoS parameter can be given by

$$
w=-1+\frac{2}{3}\left[f^{2}(\phi)+\varepsilon\right] \cdot\left(\frac{F(\phi)}{H(\phi)}\right)^{2} .
$$

Let us consider two particular cases.

\subsubsection{The case $F(\phi)=\lambda$}

In order to obtain an explicit solution using this method, one has to specify the coupling function $f(\phi)$, or put some other additional condition. Here we assume that the coupling function can be represented by

$$
f(\phi)=\sqrt{2} f 0 \sin \frac{\omega_{0}}{2} \phi
$$

With $f_{0}^{2}>1 / 2$ and $\varepsilon=-1$, the kinetic term in (17), (18) can periodically change its signature, evolving from the phantom regime to the quintessence one and vice versa. From Eqs. (21) - (24), we can find that

$$
\begin{aligned}
& \phi=\lambda t, \\
& H(\phi)=-\lambda\left[\left(f_{0}^{2}+\varepsilon\right) \phi-\frac{f_{0}^{2}}{\omega_{0}} \sin \omega_{0} \phi,\right] \\
& a=a_{0} \exp \left[-\frac{f_{0}^{2}+\varepsilon}{4} \phi^{2}+\frac{f_{0}^{2}}{2 \omega_{0}^{2}}\left(1-\cos \omega_{0} \phi\right)\right],
\end{aligned}
$$

And

$$
\begin{aligned}
& V(\phi)=\frac{3 \lambda^{2}}{2}\left[\left(f_{0}^{2}+\varepsilon\right) \phi-\frac{f_{0}^{2}}{\omega_{0}} \sin 2 \omega_{0} \phi\right]^{2} \\
& -\frac{\lambda^{2}}{2}\left[\left(f_{0}^{2}+\varepsilon\right)-f_{0}^{2} \cos \omega_{0} \phi\right] .
\end{aligned}
$$

The corresponding expression for the displacement function can be found from Eqs. (16), (26) and (27) as

$$
\beta(t)=\frac{2 \sqrt{2}}{3} \lambda f_{0} \sin \left(\frac{\omega_{0} \lambda t}{2}\right)
$$

According to Eqs. (12) And (28), we have for the EoS parameter

$$
w=-1+\frac{2}{3} \frac{\varepsilon+f_{0}^{2}\left(1-\cos \omega_{0} \lambda t\right)}{\left[\left(f_{0}^{2}+\varepsilon\right) \lambda t-f_{0}^{2} \omega_{0}^{-1} \sin \omega_{0} \lambda t\right]^{2}},
$$

And the similar expression for the deceleration parameter $q$ with 1 instead of the fraction $2 / 3$. For $\varepsilon=-1, \omega_{0}=5, f_{0}^{2}=0.56$, and $\lambda=4$, this solution is plotted in Fig. 1.

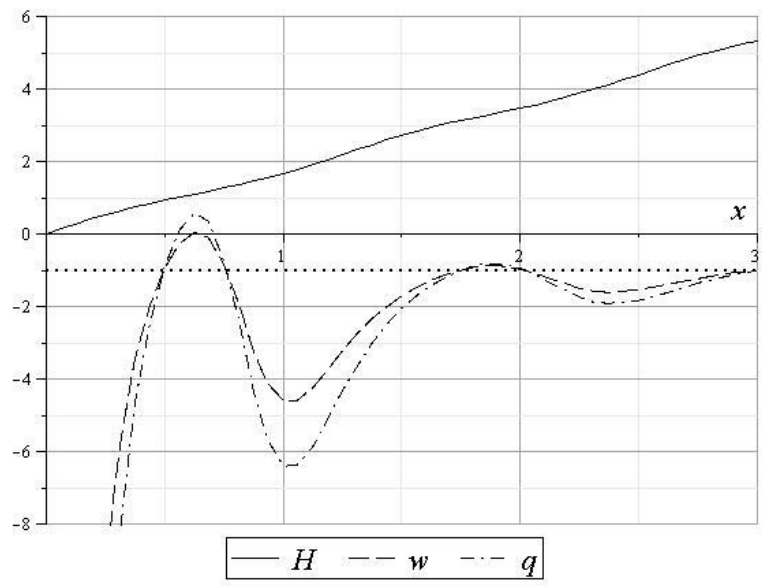

Fig. 1: The Eos Parameter $w$, the Deceleration Parameter $q$ and the Hubble Parameter $H$ in the Case $F(\phi)=\lambda$ Versus $x=\lambda t$.

It can be seen that the model expands in acceleration. At some points, where $\cos \omega_{0} \lambda t=1-f_{0}^{2}$, the expansion is the de Sitter one ( $q=-1$ ). The model periodically crosses the phantom divide in the both direction.

\subsubsection{The case $F(\phi)=\lambda \phi$}

In order to obtain an explicit solution with some interesting feature in this case, we chose the coupling function $f(\phi)$ in the following form:

$f(\phi)=\frac{f_{0}}{\phi}$.

For this choice, we have

$\phi=\phi_{0} \exp (\lambda t)$

After integration, Eqs. (21)- (24) gives

$H=-\lambda\left[f_{0}^{2} \lambda t+f_{0}^{2} \ln \phi_{0}+\varepsilon \frac{\phi_{0}^{2}}{2} \exp (2 \lambda t)\right]$,

$a(t)=a_{0} \exp \left\{-\frac{f_{0}^{2} \lambda^{2}}{2} t^{2}-f_{0}^{2} \lambda t \ln \phi_{0}-\right.$ 
$\left.-\varepsilon \frac{\phi_{0}^{2}}{4}\left(e^{2 \lambda t}-1\right)\right\}$,

Where the constant of integration is chosen so that $a_{0}=a(0)$. Besides, we can find that the potential is given by

$V(\phi)=\frac{\lambda^{2}}{2}\left[3\left(f_{0}^{2} \ln \varphi+\frac{\varepsilon}{2} \phi^{2}\right)^{2}-\varepsilon \phi^{2}-f_{0}^{2}\right]$.

After that, the EoS parameter can be found as follows

$$
w=-1+\frac{2}{3} \frac{f_{0}^{2}+\varepsilon \phi_{0}^{2} \exp (2 \lambda t)}{\left[f_{0}^{2} \lambda t+f_{0}^{2} \ln \phi_{0}+\varepsilon \frac{\phi_{0}^{2}}{2} \exp (2 \lambda t)\right]^{2}} .
$$

The similar expression can be also obtained for the deceleration parameter. Some features of this solution is shown in Fig. 2 for

$\varepsilon=-1, \phi_{0}=1$ and $f_{0}^{2}=1.12$.

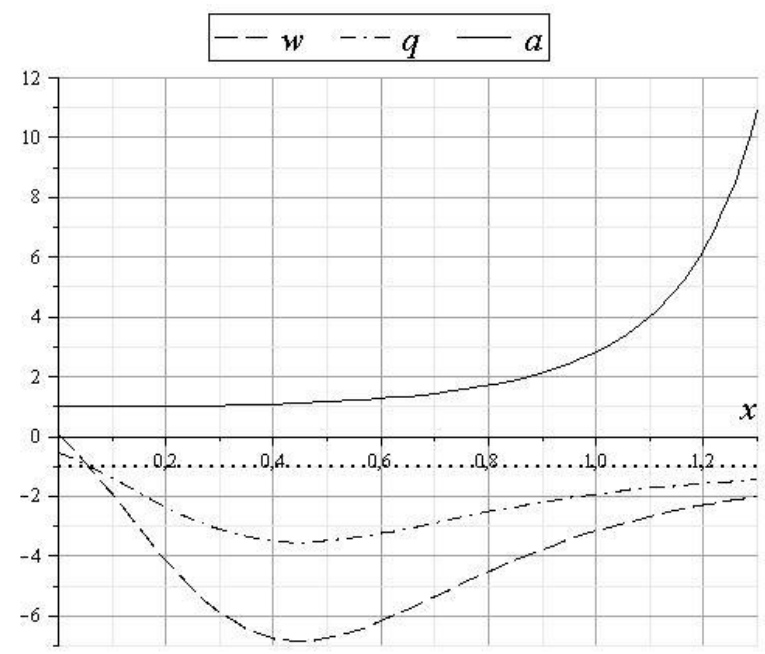

Fig. 2: The Eos Parameter $w$, the Deceleration Parameter $q$ and the Scale Factor $a$ in the Case $F(\phi)=\lambda \phi$ Versus $x=\lambda t$.

It can be seen that this model expands with acceleration as well. At the only point $t_{0}=\lambda^{-1} \ln \left(f_{0} / \phi_{0}\right)$, the expansion is de Sitter, that is $q=-1$, and the model crosses the phantom divide, evolving from the quintessence sector to the phantom one.

\subsection{The super potential method}

Some more exact solutions can be obtained in terms of the socalled superpotential. This procedure was first performed with a single scalar field by Zhuravlev and Chervon (2000), and it was later re-opened as "the first order formalism" and extended on the case of two or more fields (Bazeia et al., 2006). Keeping in mind the superpotential method, we now represent the geometrical field of displacement vector as a function of a new field $\alpha(t)$ :

$$
\beta^{2}(t)=\frac{4}{3} \dot{\alpha}^{2}(t)
$$

Now we get the new set of equations instead of (14), (15):

$$
3 H^{2}=\varepsilon \dot{\phi}^{2}+2 V(\phi)+\dot{\alpha}^{2}(t),
$$

$\dot{H}=-\varepsilon \dot{\phi}^{2}-\dot{\alpha}^{2}(t)$,

Let us introduce the superpotential function $W(\phi, \alpha)$ by the equation

$H=W(\phi, \alpha)$,

In which the Hubble parameter $H(t)$, as a function of time, is presumably expressed in terms of fields $\phi(t), \alpha(t)$. Substituting (42) into (41), one can obtain two first-order equations as follows:

$\dot{\phi}=-\varepsilon W_{\phi}, \dot{\alpha}=-W_{\alpha}$,

Where $W_{\phi}=\partial W / \partial \phi, W_{\alpha}=\partial W / \partial \alpha$. The potential can be obtained from (40), (42) and (43) as follows

$V(\phi)=\frac{3}{2} W^{2}-\frac{\varepsilon}{2} W_{\phi}^{2}-\frac{1}{2} W_{\alpha}^{2}$.

As the potential depends on $\phi$ alone, we have $\partial V / \partial \alpha=0$. Taking into account (43) and (44), it is easy to show that this is equivalent to

$\frac{d W_{\alpha}}{d t}+3 W W_{\alpha}=0$

The latter can be satisfied in many ways. Let us now consider one simple example of exact solution. We suppose that the superpotential is presented by

$W(\phi, \alpha)=X(\phi) Y(\alpha)$,

That allows to rewrite (44) in the following form:

$V(\phi)=\frac{1}{2} X^{2}(\phi)\left[3 Y^{2}(\alpha)-\varepsilon Y^{2}(\alpha)\left(\frac{X^{\prime}(\phi)}{X(\phi)}\right)^{2}-Y^{\prime 2}(\alpha)\right]$.

We consider a particular solution for this model based on

$X(\phi)=X_{0} e^{-\lambda \phi}, Y(\alpha)=\cosh (\sqrt{\mu} \alpha)$,

Where $\mu=3-\varepsilon \lambda^{2}$. So we have

$$
\begin{aligned}
& \dot{\phi}=-\varepsilon W_{\phi}=\varepsilon \lambda X_{0} e^{-\lambda \phi} \cosh (\sqrt{\mu} \alpha), \\
& \dot{\alpha}=-W_{\alpha}=-\sqrt{\mu} X_{0} e^{-\lambda \phi} \sinh (\sqrt{\mu} \alpha), \\
& H(t)=X_{0} e^{-\lambda \phi(t)} \cosh (\sqrt{\mu} \alpha(t)) .
\end{aligned}
$$

At the same time, we have the following expression for the potential (47):

$$
V(\phi)=\frac{\mu}{2} X_{0}^{2} e^{-2 \lambda \phi}
$$

Combining and integrating Eqs. (49), (50), we can get

$$
\exp (-\lambda \phi)=[\sinh (\sqrt{\mu} \alpha)]^{\varepsilon \lambda^{2} / \mu},
$$


Where the constant of integration is chosen to be equal to zero. Substituting the latter into (50), we can obtain the following equation for $\alpha$ :

$$
\dot{\alpha}=-\sqrt{\mu} X_{0}[\sinh (\sqrt{\mu} \alpha)]^{3 / \mu} .
$$

This equation can be integrated explicitly for several values of $\mu$ (or $\lambda$ and $\varepsilon$ ).

As an example, we consider the case $\varepsilon=+1, \lambda=\sqrt{3 / 2}$ or $\mu=3 / 2$. Integrating equation (54) and taking into account (39), we can obtain

$$
\alpha=\sqrt{\frac{2}{3}} \tanh ^{-1}\left[\frac{2}{3 X_{0} t+2}\right],
$$

$$
\beta^{2}=\frac{32 X_{0}^{2}}{\left[\left(3 X_{0} t+2\right)^{2}-4\right]^{2}},
$$

Where the constant of integration is chosen from the condition $\tanh \{\sqrt{3 / 2} \alpha(0)\}=1$. With the help of (51), (53) and (55), it is possible to find that

$$
H(t)=2 X_{0} \frac{3 X_{0} t+2}{\left(3 X_{0} t+2\right)^{2}-4},
$$

$a(t)=a_{0}\left[\left(3 X_{0} t+2\right)^{2}-4\right]^{1 / 3}$,

And

$$
\phi=\frac{1}{\sqrt{6}} \ln \left|\left(3 X_{0} t+2\right)^{2}-4\right|
$$

From Eqs. (12) And (56), it can be found that

$$
w=\frac{4}{\left(3 X_{0} t+2\right)^{2}}, q=\frac{1}{2}+\frac{6}{\left(3 X_{0} t+2\right)^{2}} .
$$

Time evolution of this model is plotted in Fig. 3 for $\varepsilon=+1$, $\lambda=\sqrt{3 / 2}$ and $X_{0}=3 / 2$.

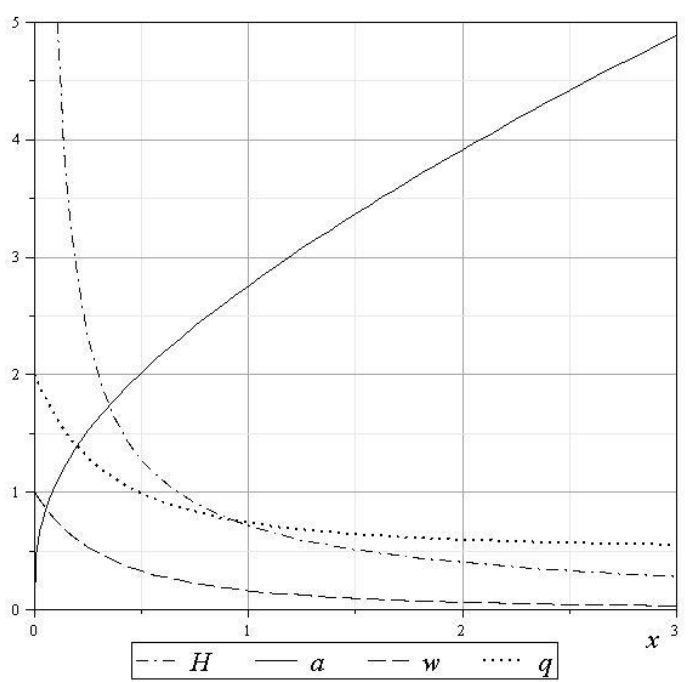

Fig. 3: The Eos Parameter $w$, the Deceleration Parameter $q$, the Scale Factor $a$ and the Hubble Parameter $H$ Versus $x=\lambda t$

\section{Lyra's cosmology of tachyon field}

In this section, we consider a tachyon field $\chi$ as a source of gravity in Lyra's cosmology. Substituting the well-known expressions of tachyonic $\rho$ and $p$ into (11), we have

$\rho_{\text {eff }}=\frac{V(\chi)}{\sqrt{1-\dot{\chi}^{2}}}+\frac{3 \beta^{2}}{8}$,

$p_{e f f}=-V(\chi) \sqrt{1-\dot{\chi}^{2}}+\frac{3 \beta^{2}}{8}$

As before, we put $H(t)=W[\chi(t), \alpha(t)]$ and suppose the substitution (39). Due to (59), the set of basic equations (8), (9) becomes as follows

$$
\begin{gathered}
\frac{3}{2} H^{2}=\frac{V(\chi)}{\sqrt{1-\dot{\chi}^{2}}}+\frac{1}{2} \dot{\alpha}^{2}, \\
\dot{H}=-\frac{V(\chi) \dot{\chi}^{2}}{\sqrt{1-\dot{\chi}^{2}}}-\dot{\alpha}^{2} .
\end{gathered}
$$

By inserting $H=W(\chi, \alpha)$ into (61), one can obtain two first-order equations,

$\dot{\chi}=-\frac{2 W_{\chi}}{3 W^{2}-W_{\alpha}^{2}}, \dot{\alpha}=-W_{\alpha}$,

Where $W_{\chi}=\partial W / \partial \chi, W_{\alpha}=\partial W / \partial \alpha$. The potential is followed from (60) - (62) in the form:

$V(\chi)=\frac{1}{2} \sqrt{\left(3 W^{2}-W_{\alpha}^{2}\right)^{2}-4 W_{\chi}^{2}}$.

As this potential is independent on $\alpha$, we have $\partial V / \partial \alpha=0$.

In view of (62), (63), it is easy to prove that the latter is equivalent to equation (45).

So we have a wide range of possibilities to solve the model Equations assuming some certain dependence $W(\chi, \alpha)$.

Instead, we can provide several classes of solution for the model evolving from some conditions on superpotential. Below, we show how it can be realized with the help of some ansatz for the superpotential.

One of the simplest ansatz for the superpotential may be written as follows:

$W_{\chi}=\lambda_{1} W^{m}(\chi, \alpha), W_{\alpha}=\lambda_{2} W^{m}(\chi, \alpha)$,

Where $m, \lambda_{1}, \lambda_{2}$ are constants, and the equality $W_{\chi, \alpha}=W_{\alpha, \chi}$ is true. Therefore in view of (45) and (64), we obtain

$H(t)=W(\chi(t), \alpha(t))=\frac{m}{3\left(t+t_{0}\right)}$,

Where $t_{0}$ is an integration constant. Hence, equations (62) become as follows 


$$
\begin{aligned}
& \dot{\chi}=-\frac{2 \lambda_{1}(m / 3)^{m-2}\left(t+t_{0}\right)^{m}}{3\left(t+t_{0}\right)^{2 m-2}-\lambda_{2}^{2}(m / 3)^{2 m-2}}, \\
& \dot{\alpha}=-\lambda_{2} \frac{(m / 3)^{m}}{\left(t+t_{0}\right)^{m}} .
\end{aligned}
$$

From the last equation in (62) and Eqs. (64), (65), it immediately follows that the displacement vector is given by

$$
\beta(t)=\lambda_{2} \frac{2}{\sqrt{3}}\left(\frac{m}{3}\right)^{m}\left(t+t_{0}\right)^{-m}
$$

The EoS parameter and the deceleration parameter (12) are constant and defined by

$$
w=-1+\frac{2}{m}, q=-1+\frac{3}{m} .
$$

In order to distinguish between various DE models, Sahni et al. (2003) proposed a cosmological diagnostic pair $\{r, s\}$ called statefinder. The statefinder test is a geometrical one based on the expansion of the scale factor $a(t)$ near the present time $t_{0}$ :

$$
\begin{aligned}
& a(t)=1+H_{0}\left(t-t_{0}\right)-\frac{1}{2} q_{0} H_{0}^{2}\left(t-t_{0}\right)^{2} \\
& +\frac{1}{6} r_{0} H_{0}^{3}\left(t-t_{0}\right)^{3}+\ldots,
\end{aligned}
$$

Where $a\left(t_{0}\right)=1$ and $H_{0}, q_{0}, r_{0}$ are the present values of the Hubble parameters, deceleration parameter and the statefinder index $r=\dddot{a} / a H^{3}$ respectively. The statefinder parameter $s$ is the combination of $r$ and $q: s=(r-1) / 3(q-1 / 2)$. The important feature of statefinder is that the spatially flat $\Lambda \mathrm{CDM}$ has the fixed point $\{r, s\}=\{1,0\}$. Departure of a DE model from this fixed point is a good way of establishing the 'distance' of this model from flat $\Lambda$ CDM. In terms of the Hubble parameter and its derivatives with respect to cosmic time the statefinder parameters of a flat FRW model are given by

$$
r=1+3 \frac{\dot{H}}{H^{2}}+\frac{\ddot{H}}{H^{3}}, \quad s=-\left(\frac{2}{3 H}\right) \frac{3 H \dot{H}+\ddot{H}}{3 H^{2}+2 \dot{H}} .
$$

With the help of Eqs. (65), (69), one can find that

$$
r=1-\frac{9}{m}+\frac{18}{m^{2}}, s=\frac{2}{m},
$$

For the model considered.

\section{Conclusion}

In this paper, we have studied FRW cosmological models in normal gauge for Lyra's manifold with the quintessence (phantom) and tachyon scalar fields as the origin of gravity. We have built the new classes of FRW cosmological models of these scalar fields in the context of Lyra's geometry. The different types of exact solution for the model are obtained by applying two procedures: the generating function method and the first-order formalism. We hope that the derived model is the next step in the development of Lyra's cosmology, and can be utilized to describe the evolutionary dynamics of the actual universe.

\section{References}

[1] Agarwal S, Pandey RK, Pradhan A (2011) LRS Bianchi type II perfect fluid cosmological models in normal gauge for Lyra's manifold. International Journal of Theoretical Physics, 50: 296-307. http://dx.doi.org/10.1007/s10773-010-0523-y.

[2] Bazeia A, Gomes C B, Losano L \& Menezes R (2006), First-order formalism and dark energy. Physics Letters B, 633: 415 http://dx.doi.org/10.1016/j.physletb.2005.12.031.

[3] Beesham A (1986), Friedmann's cosmology in Lyra's manifold. Astrophysics and Space Science, 127: 355-359. http://dx.doi.org/10.1007/BF00636548.

[4] Beesham A (1988), FLRW cosmological models in Lyra's manifold with time dependent displacement field. Australian Journal of Physics, 41: 833-842. http://dx.doi.org/10.1071/PH880833.

[5] Chimento L P, Mendez V \& Zuccala N (1999), Cosmological models arising from generalized scalar field potentials. Classical \& Quantum Gravity, 16: 3749. http://dx.doi.org/10.1088/0264-9381/16/11/319.

[6] Chaubey R (2012), Kantowski-Sachs Cosmological Model in Lyra's Geometry. International Journal of Theoretical Physics, 51:39333940. http://dx.doi.org/10.1007/s10773-012-1285-5.

[7] Diakonos FK, Saridakis EN (2009), A Statistical Solution to the Cosmological Constant Problem in the Brane world. JCAP, 0902: 030. http://dx.doi.org/10.1088/1475-7516/2009/02/030.

[8] Hoyle F, Narlikar J V (1964), a new theory of gravitation. Proceedings of the Royal Society of London Series A, 282: 191-207. http://dx.doi.org/10.1098/rspa.1964.0227.

[9] Lyra G (1951), Uber eine Modifikation der riemannschen Geometric. Mathematische Zeitschrift, http://dx.doi.org/10.1007/BF01175135.

[10] Perlmutter S, et al. (1999), Measurements of $\Omega$ and $\Lambda$ from 42 HighRedshift Supernovae. Astrophysical Journal, 517: 565. http://dx.doi.org/10.1086/307221.

[11] Pradhan A, Yadav P (2009), Accelerated Lyra's Cosmology Driven by Electromagnetic Field in Inhomogeneous Universe. International Journal of Mathematics \& Mathematical Sciences, http://dx.doi.org/10.1155/2009/471938.

[12] Pradhan A, Amirhashehi H, Zanuddin H (2011), A new class of inhomogeneous cosmological model with electromagnetic field in normal gauge for Lyra manifold. International Journal of Theoretical Physics, 50: 56-69. http://dx.doi.org/10.1007/s10773-010-0493-0.

[13] Riess AG, et al. (1998), Observational Evidence from Supernovae for an Accelerating Universe and a Cosmological Constant. Astronomical Journal, 116: 1009-1038. http://dx.doi.org/10.1086/300499.

[14] Sahni V, Sahni V, Saini T D, Starobinsky A A, Alam U (2003), Statefinder-a new geometrical diagnostic of dark energy. JETP Letters, 77 (5): 201-206. http://dx.doi.org/10.1134/1.1574831

[15] Sen D K, Dunn K A (1971), A scalar-tensor theory of gravitation in a modified Riemannian manifold. Journal of Mathematical Physics, 12: 578. http://dx.doi.org/10.1063/1.1665623.

[16] Shchigolev VK (2012), Cosmological Models with a Varying $\Lambda$ Term in Lyra's Geometry. Modern Physics Letters a, 27 (29): 1250164. http://dx.doi.org/10.1142/S0217732312501647.

[17] Shchigolev VK (2013), Cosmology with an Effective $\Lambda$-Term in Lyra Manifold. Chinese Physics Letters, 30 (11): 119801. http://dx.doi.org/10.1088/0256-307X/30/11/119801.

[18] Soleng H H (1987), Cosmologies based on Lyra's geometry. General $\begin{array}{llll}\text { Relativity \& } \quad \text { Gravitation, } & 19:\end{array}$ http://dx.doi.org/10.1007/BF00759100.

[19] Weyl H (1918), Gravitation und Elektrizität.Sitzungsber. Preuss. Akad. d. Wiss. Teil, 1: 465-480. http://www.gutenberg.org/files/43006

[20]Zhuravlev VM, Chervon SV (2000), Cosmological Inflation Models Admitting Natural Emergence to the Radiation - Dominated Stage and the Matter Domination Era. Journal of Experimental and Theoretical Physics, 91 (2): 227-238. http://dx.doi.org/10.1134/1.1311981. 